\title{
Genome Resource of Two Potato Strains of Ralstonia solanacearum Biovar 2 (Phylotype IIB Sequevar 1) and Biovar 2T (Phylotype IIB Sequevar 25) Isolated from Lowlands in Iran
}

\author{
Nasim Sedighian, ${ }^{1,2}$ Marjon Krijger, ${ }^{2}$ Tanvi Taparia, ${ }^{2,3}$ S. Mohsen Taghavi, ${ }^{1}$ Emmanuel Wicker, ${ }^{4,5}$ \\ Jan M. van der Wolf, ${ }^{2}$ and Ebrahim Osdaghi, ${ }^{1, \dagger}$ \\ ${ }^{1}$ Department of Plant Protection, College of Agriculture, Shiraz University, Shiraz 71441-65186, Iran \\ ${ }^{2}$ Business Unit Biointeractions and Plant Health, Wageningen University and Research, 6700 AA \\ Wageningen, The Netherlands \\ ${ }^{3}$ Department of Microbial Ecology, Netherlands Institute of Ecology, The Netherlands \\ ${ }^{4}$ IPME, Univ Montpellier, CIRAD, IRD, Montpellier, France \\ ${ }^{5}$ CIRAD, UMR IPME, Montpellier, France
}

\begin{abstract}
Ralstonia solanacearum, the causal agent of bacterial wilt and brown rot disease, is one of the major pathogens of solanaceous crops, including potato, around the globe. Biovar $2 \mathrm{~T}$ (phylotype II/sequevar 25) of $R$. solanacearum is adapted to tropical lowlands and is only reported in South America and Iran. Thus far, no genome resource of the biovar 2T of the pathogen has been available. Here, we present the near-complete genome sequences of the biovar 2T strain CFBP 8697 as well as strain CFBP 8695 belonging to biovar 2 race 3, both isolated from potato in Iran. The genomic data of biovar $2 \mathrm{~T}$ will extend our understanding of the virulence features of $R$. solanacearum and pave the way for research on biovar $2 T$ functional and interaction genetics.
\end{abstract}

\section{Genome Announcement}

Bacterial wilt and brown rot disease caused by Ralstonia solanacearum species complex (RSSC) is one of the economically important constraints of potato (Solanum tuberosum) production around the globe. RSSC strains possess a wide range of phylogenetic, biologic, and host-range diversity, leading to subdivision of the pathogen into several phylotypes and sequevars, biovars, and races based on the genomic feature, biological activity, and host range of the strains, respectively (Buddenhagen et al. 1962; Prior and Fegan 2005; Wicker et al. 2012). Comprehensive polyphasic investigations resulted in the classification of the RSSC into five races, six biovars, and four phylotypes (Castillo and Greenberg 2007; Fegan and Prior 2005; Hayward 1964, 1994). Biovar 2 strains were further divided into two phenotypes 2A and 2T (Marin and El-Nashar 1993). Biovar 2T strains are known to cause bacterial wilt of potato in lowlands and tropical geographic areas and are considered the leaststudied member within the RSSC, with no information of the genomic features and virulence repertoires. Biovar 2T strains were isolated from potato and eucalyptus (Marques et al. 2012) and are found in three of the four phylotypes (i.e., phylotypes II, III, and IV) within the RSSC

${ }^{\dagger}$ Corresponding author: E. Osdaghi; eosdaghi@shirazu.ac.ir

The author(s) declare no conflict of interest.

Accepted for publication 2 April 2020.

(C) 2020 The American Phytopathological Society

\section{Funding}

This study was financially supported by Wageningen University and Research (The Netherlands) and Shiraz University (Iran). N. Sedighian benefited from the sabbatical interactions promoted and supported by funding from the Ministry of Science, Research and Technology, Iran.

\section{Keywords}

bacterial wilt, biovar/race, genomics, Ralstonia solanacearum species complex 
(Fegan and Prior 2005). Phylotype III biovar 2T strains were isolated in Africa, while phylotype IV biovar 2T strains were isolated in the Philippines, Japan, and Indonesia (Fegan and Prior 2005). Phylotype IIB biovar 2T strains, which correspond to sequevars 25 to 28 reported by Fegan and Prior (2005), were isolated in Brazil (Costa et al. 2007; Santana et al. 2012), French Guyana (Deberdt et al. 2014), and southwestern Iran from potato and tomato (Nouri et al. 2009).

Here, we present the complete genome sequence of the biovar 2T (phylotype IIB sequevar 25) strain Sh16 = CFBP 8697 of $R$. solanacearum, isolated from potato in tropical lowlands of southwestern Iran in 2002, as well as a potato brown rot ecotype (phylotype IIB sequevar 1) strain K2 = CFBP 8695, isolated from the same host in western Iran in 2017. Pathogenicity, host range, and phylogenetic status of the strains using a five-gene (i.e., egl, fliC, gyrB, mutS, and $r p / B$ ) multilocus sequence analysis were reported previously (Sedighian 2020).

Strains CFBP 8695 and CFBP 8697 were grown on yeast-extract peptone glucose agar (YPGA) medium as described previously (Osdaghi et al. 2018), and DNAs were extracted using the Wizard genomic DNA purification kit according to the recommendation of the manufacturer (Promega, Madison WI, U.S.A.). The quality and quantity of the DNAs were spectrophotometrically evaluated and were adjusted to 1,500 to $2,000 \mathrm{ng} \mathrm{ll}^{-1}$ using the Nanodrop ND-100 (Nanodrop Technologies, Waltham, MA, U.S.A.). DNA was sequenced using Illumina NovaSeq technologies (BaseClear B.V., Leiden, The Netherlands), and shotgun sequencing yielded 150-bp paired-end reads. Quality control and downstream processing of the whole genome sequence data were performed on CLC Genomics Workbench 12.0.2 (Qiagen N.V., Hilden, Germany). Trim Reads v. 2.3 was used to perform quality trimming on the whole-genome sequences. Parameters included a quality limit of 0.05 , ambiguous limit of 2, minimum nucleotide length of 45 , and automatic read-through adapter trimming. Furthermore, Map Reads to Contigs v. 1.3 was used to perform read mapping with a match score of 1 , mismatch cost of 2 , insertion cost of 3 , deletion cost of 3 , length fraction of 0.8 , and similarity fraction of 0.8 . Nonspecific matches were mapped randomly. De Novo Assembly 1.5 was used to perform de novo assembly and update the contigs with the minimum contig length of 500 , length fraction of 0.8 , and similarity fraction of 0.8 , automatic word and bubble size, and scaffolding. Finally, Extract Consensus Sequence v. 0.6 added annotation (conflicts, indels, low coverage), 'N' ambiguity symbols, and voted for conflict resolution in order to extract the final contig sequences.

The genome of $R$. solanacearum is organized into two large circular replicons, including the chromosome, which is the larger replicon, carrying the majority of the housekeeping genes, and megaplasmid, which is the smaller replicon, carrying genes involved in bacterial adaptation to the environment and plant host (Salanoubat et al. 2002). Hence, the obtained reads were mapped onto the chromosome of the reference strain IPO1609 (NZ_LN651282) and its megaplasmid (NZ_LN651281) with a length fraction of 0.8 and similarity fraction of 0.8 , without masking, to update the contigs from de-novo assembly. Nonspecific matches were mapped randomly. Regions of contigs where no reads mapped were removed from the assembly. From the mapping of the contigs to the references, two consensus sequences were extracted for the chromosome and the megaplasmid. Subsequently, genome annotation was performed using the GeneMark S+ (v 4.6) suite implemented in the National Center for Biotechnology Information (NCBI) Prokaryotic Genome Annotation Pipeline with default settings (Borodovsky and Lomsadze 2014). For each strain, genome length (base pairs), $\mathrm{G}+\mathrm{C}$ content (\%), total number of protein-coding genes, RNA genes, and pseudogenes are summarized in Table 1.

A total of $18,712,784$ reads were generated for strain CFBP 8697, after quality control. De-novo assembly of the trimmed reads led to 79 contigs. When aligned against the reference genome IPO1609, 10,407,265 reads mapped to its chromosome and 5,868,984 reads mapped to its megaplasmid. For strain CFBP 8695, 24,781,054 reads were generated post trimming, leading to 152 contigs in de-novo assembly, while 15,571,786 and 8,341,567 reads mapped to its chromosome and megaplasmid, respectively.

The chromosome and megaplasmid replicons of strain CFBP 8697 were calculated as $3,142,020$ and $1,748,866 \mathrm{bp}$, respectively; while the length of the replicons in strain CFBP 8695 was $3,299,152$ and $1,814,285$ bp, respectively. Total genome length for these two strains, thus, ranges from 4.891 to $5.11 \mathrm{Mb}$. Average nucleotide identity (ANI) was calculated using a combination of three online services, i.e., JSpeciesWS (Richter et al. 2016), ANI calculator (Rodriguez-R and Konstantinidis 2016), and OrthoANlu (Yoon et al. 2017), using the previously described procedure (Osdaghi et al. 2020). The ANI value between strains CFBP 8697 and CFBP 8695 was calculated as $99.5 \%$, while the ANI value between the latter

Vol. 33, No. 7, 2020 / 873 
Table 1. Genome information for the Ralstonia solanacearum strains sequenced in this study ${ }^{\mathrm{a}}$

\begin{tabular}{|c|c|c|c|c|}
\hline \multirow[b]{2}{*}{ Genomic features } & \multicolumn{2}{|c|}{ CFBP $8697=$ Sh16 } & \multicolumn{2}{|c|}{ CFBP $8695=$ K2 } \\
\hline & Chromosome & Megaplasmid & Chromosome & Megaplasmid \\
\hline Taxonomic status & \multicolumn{2}{|c|}{ Phylotype IIB sequevar 25} & \multicolumn{2}{|c|}{ Phylotype IIB sequevar 1} \\
\hline $\begin{array}{l}\text { GenBank accession } \\
\text { number }\end{array}$ & CP047136 & CP047137 & CP047138 & СР047139 \\
\hline Genome length (bp) & $3,142,020$ & $1,748,866$ & $3,299,152$ & $1,814,285$ \\
\hline$G+C$ content $(\%)$ & 67.22 & 67.31 & 66.73 & 66.96 \\
\hline Genome coverage & $400 X$ & $400 X$ & $600 X$ & $600 X$ \\
\hline \multicolumn{5}{|l|}{ Number of } \\
\hline Contig(s) & \multicolumn{2}{|c|}{79} & \multicolumn{2}{|c|}{152} \\
\hline Genes (total) & \multicolumn{2}{|c|}{4,309} & \multicolumn{2}{|c|}{4,572} \\
\hline CDSs (total) & \multicolumn{2}{|c|}{4,274} & \multicolumn{2}{|c|}{4,536} \\
\hline Genes (coding) & \multirow{2}{*}{\multicolumn{2}{|c|}{4,025}} & \multicolumn{2}{|c|}{4,204} \\
\hline CDSs (with protein) & & & \multicolumn{2}{|c|}{4,204} \\
\hline RNAs & \multicolumn{2}{|c|}{35} & \multicolumn{2}{|c|}{$\begin{array}{c}4,204 \\
36\end{array}$} \\
\hline rRNAs (5S, 16S, 23S) & \multicolumn{2}{|c|}{$2,1,1$} & \multicolumn{2}{|c|}{$2,1,1$} \\
\hline tRNAs & \multirow{2}{*}{\multicolumn{2}{|c|}{$\begin{array}{c}28 \\
3\end{array}$}} & \multicolumn{2}{|c|}{28} \\
\hline ncRNAs & & & & \\
\hline Pseudogenes & \multicolumn{2}{|c|}{249} & \multicolumn{2}{|c|}{332} \\
\hline Type III effectors (T3Es) & 21 & 37 & 21 & 37 \\
\hline $\mathrm{T}^{2} \mathrm{Es}^{\mathrm{b}}$ & $\begin{array}{l}\text { RipA5, RipB, RipE1, } \\
\text { RipG4, RipG5, RipG6, } \\
\text { RipG7, RipH1, Ripl,*} \\
\text { RipM, RipS1, RipS5, } \\
\text { RipV1, RipV2, RipW, } \\
\text { RipY, RipAA, }{ }^{*} \text { RipAE, } \\
\text { RipAJ, RipAM, RipAX1 }\end{array}$ & $\begin{array}{l}\text { RipA2, RipA3, }{ }^{*} \text { RipA4, } \\
\text { RipA5, RipC1, RipD, } \\
\text { RipE2, RipF1, RipF2, } \\
\text { RipG2, RipG3, RipH2, } \\
\text { RipH3, RipN, RipO1, } \\
\text { RipQ, RipR, RipS2, } \\
\text { RipS3, RipS7, RipU, } \\
\text { RipX, RipZ, RipAB, } \\
\text { RipAC, RipAD, RipAl, } \\
\text { RipAN, RipAO, RipAP, } \\
\text { RipAQ, RipAR, RipAS, } \\
\text { RipAT, RipAY, RipBI, } \\
\text { RipBH }\end{array}$ & $\begin{array}{l}\text { RipA5, RipB, RipE1, } \\
\text { RipG4, RipG5, RipG6, } \\
\text { RipG7, RipH1, Ripl, } \\
\text { RipM, RipS1, RipS5, } \\
\text { RipV1, RipV2, RipW, } \\
\text { RipY, RipAA, RipAE, } \\
\text { RipAJ, RipAM, RipAX1 }\end{array}$ & $\begin{array}{l}\text { RipA2, RipA3,* RipA4, } \\
\text { RipA5, RipC1, RipD, } \\
\text { RipE2, RipF1, RipF2, } \\
\text { RipG2, RipG3, RipH2, } \\
\text { RipH3, RipN, RipO1, } \\
\text { RipQ, RipR, RipS2, } \\
\text { RipS3, RipS7, RipU, } \\
\text { RipX, RipZ, RipAB, } \\
\text { RipAC, RipAD, RipAI, } \\
\text { RipAN, RipAO, RipAP, } \\
\text { RipAQ, RipAR, RipAS, } \\
\text { RipAT, RipAY, RipBH, } \\
\text { RipBI }\end{array}$ \\
\hline
\end{tabular}

a CFBP = International Center for Microbial Resources-French Collection for Plant-Associated Bacteria; CDS = coding sequence.

${ }^{b}$ Asterisks ( $\left.{ }^{*}\right)$ indicate only a pseudogene copy of the effector is present in the strain.

two strains and reference strain IPO1609 was 99.37 and $99.91 \%$, respectively, and $92 \%$ with the reference strain GMI1000. These ANI indices confirmed the close phylogenetic relationships between the phylotype IIB biovar 2T (phylotype IIB sequevar 25) clade and those of the potato brown rot ecotype (phylotype IIB sequevar 1), as reported previously (Wicker et al. 2012). In order to determine the type III effector (T3E) gene set of the strains sequenced in this study, we used the Ralsto T3E online service according to the procedure described by Peeters et al. (2013), and the obtained results are summarized in Table 1.

The genome projects announced here have been deposited at DDBJ/EMBL/GenBank under the accession numbers shown in Table 1. For all the sequences, the first version of the accession numbers is described in this paper. A pure culture of both strains sequenced in this study are available from the French International Center for Microbial Resources collection of plant-associated bacteria.

\section{Data Accessibility}

The data that support the findings of this study are available in the NCBI GenBank database.

\section{Author-Recommended Internet Resources}

French International Center for Microbial Resources collection of plant-associated bacteria: http://www6.inra.fr/cirm_eng/CFBP-Plant-Associated-Bacteria

Ralsto T3E service: https://iant.toulouse.inra.fr/bacteria/annotation/site/prijT3Ev3 


\section{Literature Cited}

Borodovsky, M., and Lomsadze, A. 2014. Gene identification in prokaryotic genomes, phages, metagenomes, and EST sequences with GeneMarkS suite. Curr. Protoc. Microbiol. 32:7.

Buddenhagen, I., Sequeira, L., and Kelman, A. 1962. Designation of races in Pseudomonas solanacearum. Phytopathology 52:726.

Castillo, J. A., and Greenberg, J. T. 2007. Evolutionary dynamics of Ralstonia solanacearum. Appl. Environ. Microbiol. 73:1225-1238.

Costa, S. B., Ferreira, M. A. S. V., and Lopes, C. A. 2007. Diversidade patogênica e molecular de Ralstonia solanacearum da região Amazonica Brasileira. Fitopatol. Bras. 32:285-294.

Deberdt, P., Guyot, J., Coranson-Beaudu, R., Launay, J., Noreskal, M., Rivière, P., Vigné, F., Laplace, D., Lebreton, L., and Wicker, E. 2014. Diversity of Ralstonia solanacearum in French Guiana expands knowledge of the "emerging ecotype". Phytopathology 104:586-596.

Fegan, M., and Prior, P. 2005. How complex is the Ralstonia solanacearum species complex? Pages 449-461 in: Bacterial wilt disease and the Ralstonia solanacearum species complex. C. Allen, P. Prior, and A. C. Hayward, eds. American Phytopathological Society Press, St. Paul, MN, U.S.A.

Hayward, A. C. 1964. Characteristics of Pseudomonas solanacearum. J. Appl. Bacteriol. 27:265-277.

Hayward, A. C. 1994. Systematics and phylogeny of Pseudomonas solanacearum and related bacteria. Pages 123-135 in: Bacterial wilt: the disease and its causative agent, Pseudomonas solanacearum. Hayward, A. C., and Hartman, G. L., eds. CAB International, Wallingford, U.K.

Marin, J. E., and El-Nashar, H. M. 1993. Pathogenicity of the new phenotypes of Pseudomonas solanacearum from Perú. Pages 78-84 in: Bacterial Wilt ACIAR Proceedings No. 45. Proceedings of the International Conference at Kaohsiung, Taiwan, October 28-31, 1992. G. L. Hartman and A. C. Hayward, eds. Australian Centre for International Agricultural Research, Canberra, Australia.

Marques, E., Uesugi, C. H., Ferreira, M. A., and Rezende, D. V. 2012. Characterization of isolates of Ralstonia solanacearum biovar 2, pathogenic to Eucalyptus "urograndis" hybrids. Trop. Plant Pathol. 37:399-408.

Nouri, S., Bahar, M., and Fegan, M. 2009. Diversity of Ralstonia solanacearum causing potato bacterial wilt in Iran and the first record of phylotype I//biovar $2 T$ strains outside South America. Plant Pathol. 58:243-249.
Osdaghi, E., Portier, P., Briand, M., Taghouti, G., and Jacques, M.-A. 2018. Draft genome sequences of the type strains of three Clavibacter subspecies and atypical peach-colored strains isolated from tomato. Microbiol Resour Announc 7:e01357-e18.

Osdaghi, E., Rahimi, T., Taghavi, S. M., Ansari, M., Zarei, S., Portier, P., Briand, M., and Jacques, M. A. 2020. Comparative genomics and phylogenetic analyses suggest several novel species within the genus Clavibacter, including nonpathogenic tomato-associated strains. Appl. Environ. Microbiol. 86:e02873-e19.

Peeters, N., Carrère, S., Anisimova, M., Plener, L., Cazalé, A. C., and Genin, S. 2013. Repertoire, unified nomenclature and evolution of the type III effector gene set in the Ralstonia solanacearum species complex. BMC Genomics 14:859.

Prior, P., and Fegan, M. 2005. Recent developments in the phylogeny and classification of Ralstonia solanacearum. Acta Hortic.: 127-136.

Richter, M., Rosselló-Móra, R., Oliver Glöckner, F., and Peplies, J. 2016. JSpeciesWS: A web server for prokaryotic species circumscription based on pairwise genome comparison. Bioinformatics 32:929-931.

Rodriguez-R, L. M., and Konstantinidis, K. T. 2016. The enveomics collection: A toolbox for specialized analyses of microbial genomes and metagenomes. PeerJ. 4: e1900:v1. Published online. https://doi.org/10.7287/peerj.preprints.1900v1.

Salanoubat, M., Genin, S., Artiguenave, F., Gouzy, J., Mangenot, S., Arlat, M., Billault, A., Brottier, P., Camus, J. C., Cattolico, L., Chandler, M., Choisne, N., Claudel-Renard, C., Cunnac, S., Demange, N., Gaspin, C., Lavie, M., Moisan, A., Robert, C., Saurin, W., Schiex, T., Siguier, P., Thébault, P., Whalen, M., Wincker, P., Levy, M., Weissenbach, J., and Boucher, C. A. 2002. Genome sequence of the plant pathogen Ralstonia solanacearum. Nature 415:497-502.

Santana, B. G., Lopes, C. A., Alvarez, E., Barreto, C. C., Allen, C., and Quirino, B. F. 2012. Diversity of Brazilian biovar 2 strains of Ralstonia solanacearum. J. Gen. Plant Pathol. 78:190-200.

Sedighian, N. 2020. Phylogeny, population genetics, and pathogenicity of Ralstonia solanacearum in Iran. Ph.D. thesis. Shiraz University, Iran.

Wicker, E., Lefeuvre, P., de Cambiaire, J. C., Lemaire, C., Poussier, S., and Prior, P. 2012. Contrasting recombination patterns and demographic histories of the plant pathogen Ralstonia solanacearum inferred from MLSA. ISME J. 6:961-974.

Yoon, S. H., Ha, S. M., Lim, J., Kwon, S., and Chun, J. 2017. A large-scale evaluation of algorithms to calculate average nucleotide identity. Antonie van Leeuwenhoek 110:1281-1286. 